\title{
An Investigation of the Adaptive Coefficient Setting Method for the Two-Hop Consensus Protocol and the Effect of Network Topology on Power Systems
}

\author{
Craig Wang
}

\begin{abstract}
In multi-agent systems, the consensus protocol is a distributed algorithm that instructs the information exchange between neighbouring agents in order to reach a consensus state for all of the agents within the network. Consensus problems have an extensive range of applications, including formation control problems (mobile robots, unmanned aerial vehicles, and automated highway systems), flocking, coupled oscillators, and payload transportation. In this report, we provide a brief review of consensus protocols and their applications. We propose an adaptive coefficient setting method for two-hop relay protocol, and analyse it in comparison with the Metropolis method for nearest neighbour protocol. The simulation shows that there is a significant improvement on the consensus speed thus the coefficient setting method can be more efficiently used in these applications. We also evaluate the effect of network topology on the power systems.
\end{abstract}

\section{Introduction}

Consensus problems generally involve information exchange between agents within the network; a common group objective is reached through agents interacting with each other. These problems cover many disciplines. For example, biologists use them to study the collective behaviour of animals such as ants, birds and fish, known as flocking [1]; physicists use them to study synchronization of coupled oscillators [2], which could increase the performance of oscillator networks; the engineers implement the theory to solve 
real world problems such as Smart Grid, a modernization of the electricity grid, that can significantly increase the efficiency of electricity distribution; yet most theoretical analyses have so far relied on matrix and graph theory.

A consensus protocol is a distributed method of obtaining global information or achieving a common group objective by enabling communication between agents in the system. Distributed methods are more suitable for large networks than centralised methods, as they enable better performance and faster operation. Another advantage is that they are able to increase the ability of the system to survive single point failures, therefore creating a more robust system. The protocols can also be designed specifically for different applications due to their versatility.

In this report, we further investigate consensus protocols. Firstly, we outline preliminary knowledge and notation on matrix theory and graph theory. Secondly, we present a discussion on different forms of consensus protocols that currently exist, such as switching topology versus fixed topology and nearest neighbour versus multi-hop protocol. We also outline some of the important applications of these protocols, including Smart Grid, flocking, and synchronization of coupled oscillators.

In the second part of the report, we investigate two main problems. Firstly, the effect of network topology on consensus speed, specifically tree, ring, star, two-hop and complete topologies. Secondly, an investigation of two-hop relay (second-order neighbour) protocol performance. We briefly introduce the twohop consensus protocol which has previously been discussed in [3] and [4]. Since there are no explicit descriptions about the coefficients, we fill in this gap by proposing an adaptive coefficient setting method for the two-hop consensus, which leads to the key contribution of this paper. We provide simulation results followed by discussions on the consensus process in comparison with the existing method. At last, we will conclude our project and propose some future research directions.

The goals of this project are to understand the basic concepts of consensus protocols, their applications, and to investigate different ways of increasing the consensus speed. This investigation was motivated by Smart Grid and other power system applications. Ideally this project will set the foundation for the future research in the field of Consensus Protocols. 


\section{Preliminaries}

\section{Graph theory}

The network topology for a group of agents is represented as a graph, $G=(V$, $E)$, where $v_{i} \epsilon V$ is the set of nodes where $i=1,2 \ldots n$, and $e_{i j}=\left(v_{i}, v_{j}\right) \in E$ is the set of edges joining agents of index $i$ and $j . v_{i}$ and $v_{j}$ are neighbours of each other if there exists $e=\left(v_{i}, v_{j}\right)$, the first order neighbours of agent $i$ is denoted as $N_{i}=\left\{v_{j} \epsilon V \mid\left(v_{i}, v_{j}\right) \in E\right\}$. The second order neighbours of agent $\mathrm{i}$ is denoted as $N i^{2}=\left\{v_{k} \in V \mid v_{k} \Theta N_{j^{\prime}} v_{j} \in N_{i^{\prime}}, k \neq i\right.$ and $\left.k \neq j\right\}$.

A graph is undirected if eij $\boldsymbol{\epsilon} E$ implies eji $\boldsymbol{\epsilon} E$. Otherwise the graph is directed. A complete graph is when all the nodes are neighbours of each other. The degree of a node is denoted by $\left|N_{i}\right|$, which is the total number of first order neighbours, the out-degree of $v_{i}$ is the number of edges $e_{i j}=\left(v_{i^{\prime}} v_{j}\right)$.

\section{Matrix theory}

The adjacency matrix $A=\left[a_{i j}\right] € R^{n \times n}$ of graph $\mathrm{G}$ of $\mathrm{n}$ agents is defined by:

$a_{i j}= \begin{cases}1, & v_{j} \in N_{i} \\ 0, & \text { otherwise }\end{cases}$

The diagonal matrix $D=\left[d_{i j}\right] \in R^{n \times n}$ of graph $\mathrm{G}$ of $\mathrm{n}$ agents is defined by:

$d_{i j}=\left\{\begin{array}{lr}0 & \text { otherwise } \\ \left|N_{i}\right| & i=j\end{array}\right.$

The Laplacian $L=\left[l_{i j}\right] \epsilon R^{n \times n}$ of graph $\mathrm{G}$ of $\mathrm{n}$ agents is given by $L=D-A$, such that

$l_{i j}=\left\{\begin{aligned}-1, & v_{j} \in N_{i} \\ \left|N_{i}\right|, & j=i \\ 0, & \text { otherwise }\end{aligned}\right.$

Since both the row sum and column sum of a graph Laplacian is 0 , the Laplacian matrix always has a zero eigenvalue $\lambda_{1}=0$, and it has eigenvalues of $0=\lambda_{1} \leq \lambda_{2} \leq \lambda_{3} \ldots \leq \lambda_{n^{\prime}}$ where the second smallest eigenvalue $\lambda_{2}$ is called the algebraic connectivity of a graph.

\section{Discussion on consensus protocol}

Consensus problems involve an information exchange process, in which agents within the system communicate with each other via a pre-determined consensus protocol. A consensus, i.e. a common value or objective is reached after certain 
time or certain iterations. It was first discussed in computer science, now it has found its way into many other areas including biology, physics and engineering. We will focus on the multi-agent coordination aspects of consensus problems.

A continuous time linear consensus protocol is described in [6-9], and widely used in other research papers, the dynamics are described as: $\dot{x}_{i}=\sum_{j \in N i} a_{i j}\left(x_{j}-x_{i}\right)$, where $x_{i}$ represents the information state of the ith agent, $\dot{x}_{i}$ represents the rate of change of $x_{i}$ and $a_{i j}$ is the adjacency matrix cell associated with the edge $\left(v_{i^{\prime}}, v_{j}\right)$. In other papers $[3,5], a_{i j}$ is treated as the weighted adjacency matrix, where $0<a_{i j}<1$ if $v_{i}$ and $v_{j}$ are neighbours, otherwise $a_{i j}=0$. This protocol can also be written as $\dot{x}=-L x$, where $L$ is the graph laplacian.

The corresponding discrete time protocol is $x_{i}^{k+1}=x_{i}^{k}+\sum_{j \in N i} a_{i j}\left(x_{j}^{k}-x_{i}^{k}\right)$, where $x_{j}^{k}$ represents the information state of the $\mathrm{i}^{\text {th }}$ agent at the kth iteration. This protocol can be represented in the matrix form as $X^{k+1}=P_{\epsilon} X^{k}$, where $P_{\epsilon}$ is a nonnegative stochastic matrix as described [7] and [9]. Later on in [5] and [10], the protocol has an alternate form of $X^{k+1}=D X^{k}$, where $\mathrm{D}$ is also a stochastic matrix but may have negative entries, defined as the following:

$$
D=\left[\begin{array}{ccccc}
1-\sum_{j \in N_{i}} a_{1 j} & \ldots & a_{1 i} & \ldots & a_{1 n} \\
\cdots & \ldots & \ldots & \ldots & \ldots \\
a_{11} & \ldots & 1-\sum_{j \in N_{i}} a_{i j} & \ldots & a_{i n} \\
\ldots & \ldots & \ldots & \ldots \\
a_{n 1} & \ldots & a_{n i} & \ldots & 1-\sum_{j \in N_{n !}} a_{n j}
\end{array}\right]
$$

Where $a_{i j}$ is weighted entries of the adjacency matrix.

In order to conduct the stability analysis of the protocol, the matrix $P_{\epsilon}$ or $D$ needs to satisfy the following conditions: 1. The sums of rows and columns are all ones; 2 . The eigenvalues $\left|\lambda_{i}\right|<1$, for $\mathrm{i}=2, \ldots, \mathrm{n}$. Then by defining a positive definite Lyapunov function, it is proven in $[5,9,10]$ that the protocol is asymptotically stable and they would reach a consensus value of the average initial value, which is $x_{e q}=1 / n \sum_{i=1}^{n} x_{i}^{0}$. However it would take too long to reach an exact consensus, therefore a terminating condition is usually required. For distributed computation, the terminating condition should also be a function of local values only. An example of such a condition is described in [10] $\sum_{l=k-L}^{k} \mid x_{i}^{l}$ $-x_{i}^{l-1} \mid<\boldsymbol{\epsilon}$, where $\mathrm{L}$ is the number of consecutive iterations to investigate, and $\boldsymbol{\epsilon}$ is a small number that represents the error.

\section{Applications}

There are many applications related to the consensus protocols, multi-agent coordination is particularly appealing and it attracts many researchers to investigate. 
1. The key application we are looking at in this paper is Smart Grid, a digitally enabled electrical grid that gathers, distributes and transfers electricity intelligently. It consists of millions of automated electronic meters that will be connected to servers which manage the grid. It can achieve better reliability, better performance and more efficiency compared to a conventional grid which was still based on Tesla's design in 1888. The largest existing Smart Grid is built in Italy, and brings an annual saving of 500 million euros[11]. The Smart Grid could collect global information distributedly using consensus protocols, and it is able to analyse the information in order to perform control actions, such as load shedding and load restoration.

2. Flocking is a naturally occurring phenomenon, also known as swarming behaviour in insects. Scientist and engineers have studied this type of behaviour and applied it in distributed sensing using mobile sensor networks, automated parallel delivery of payloads and performing military missions such as application in unmanned aerial vehicles (UAVs). There are three flocking rules introduced by Reynolds in [12]: 1. Flock centering, where individual vehicles attempt to stay close to nearby flock mates; 2. Collision avoidance, avoid collisions with nearby flock mates; 3. Velocity matching, attempt to match velocity with nearby flock mates. The role of consensus protocol is for an agent to achieve the matching velocity with respect to its neighbours. A relatively advanced protocol for flocking is described in [1]:

$$
\begin{gathered}
\dot{x}_{i}=u_{i}=\sum_{j \in N_{i}} \varphi_{\alpha}\left(\left\|q_{j}-q_{i}\right\|_{\alpha}\right) n_{i j}+\sum_{j \in N_{i}} a_{i j}\left(p_{j}(t)-p_{i}(t)\right) \\
+f_{i}^{\gamma}\left(q_{i}, p_{i}, q_{r}, p_{r}\right)
\end{gathered}
$$

It can be easily spotted that the second term of the sum is a consensus term.

3. The synchronization of coupled oscillators attracts attention from many scientists in the field of biology to study networks of coupled neurons. Neural oscillation is a repetitive activity in the central nervous system. This process is driven by a mechanism located within individual neurons or by interaction between neurons. Their functionality is not yet fully understood, which make them even more interesting. One model of coupled oscillators on a graph is described in [13]

$\dot{\theta}_{i}=\kappa \sum_{j \in N_{i}} \sin \left(\theta_{j}-\theta_{i}\right)+\omega_{i}$

Where $\theta_{i}$ and $\omega_{i}$ are the phase and frequency of the $i^{\text {th }}$ oscillator. This dynamic is a nonlinear extension of the continuous consensus protocol. 
Other applications include vehicle formation control, which can direct microsatellite clusters to form a precise space-telescope or a mapping-array; distributed sensor fusion in sensor networks, which is posing various distributed consensus to implement the Kalman filter [14], or a linear least-squares estimator.

\section{Coefficient setting methods ${ }^{1}$}

By choosing a coefficient setting method, we can uniquely define the matrix D in the updating scheme, which would be used in our simulation. There are a number of such methods. For a simple consensus protocol, the coefficient $a_{i j}$ in the equation is originally set by the Uniform method, where $\mathrm{N}$ is the number of nodes during normal operation. It works optimally (can reach consensus in a single step) when the graph is complete.

Uniform Method: $a_{i j}= \begin{cases}\frac{1}{N} & v_{j} \in N_{i} \\ -\sum_{j \in N_{i}} \frac{1}{N} & i=j \\ 0 & \text { otherwise }\end{cases}$

However, there are rarely any complete graph topologies in real world applications, since the communication cost is too much. Later on, the Metropolis method was proposed in [15].

Metropolis Method : $a_{i j}= \begin{cases}\frac{1}{\max \left(N_{i}, N_{j}\right)+1} & v_{j} \in N_{i} \\ -\sum_{j \in N_{i} \frac{1}{\max \left(N_{i}, N_{j}\right)+1}} & i=j \\ 0 \quad \text { otherwise }\end{cases}$

It is verified that the Metropolis method could guarantee the convergence of information, while it adaptively configured the coefficient based on the information of local agents $v_{i}$ and $v_{j}$. This method shows some performance improvement compared to the Uniform method. Another improvement is the Mean Metropolis method proposed in [5]. This is based on the stability analysis of the consensus protocol with some modifications. In the stability analysis, we have the inequality $a_{i j}<2 / / \mathrm{N}_{i}+N_{j}$ to guarantee the convergence. The convergence condition is satisfied by adding $\boldsymbol{\epsilon}$, which is a small positive number to the denominator.

Mean Metropolis Method: $a_{i j}= \begin{cases}\frac{2}{N_{i}+N_{j}+\epsilon} & v_{j} \in N_{i} \\ -\sum_{j \in N_{i}} \frac{2}{N_{i}+N_{i}+\epsilon} & i=j \\ 0 & \text { otherwise }\end{cases}$

1 In this and the following sections, we let $N_{i}$ represent the number of first order neighbours for the $\mathrm{i}^{\text {th }}$ node. $N_{i}^{2}$ represent the number of second order neighbours for the $\mathrm{i}^{\text {th }}$ node. 
[5] compared the performance of those coefficient setting methods, and it is shown that the Mean Metropolis method could achieve near optimal consensus speed. Inspired by the listed coefficient setting methods, we will propose a method involving second-order neighbour information.

The three methods described above are all distributed methods, such that the coefficient can be determined without any global information. There also exists a centralised method, called Particle Swarm Optimization (PSO)[16]. This method can obtain the optimal coefficient for a particular configuration, however it can only be implemented offline, requires global information, and can be time consuming, therefore should not be used in real-time applications. Nevertheless it can be used as a reference in the simulations to compare with other coefficient setting methods.

\section{Proposal of the Coefficient Setting Method for Two- Hop consensus}

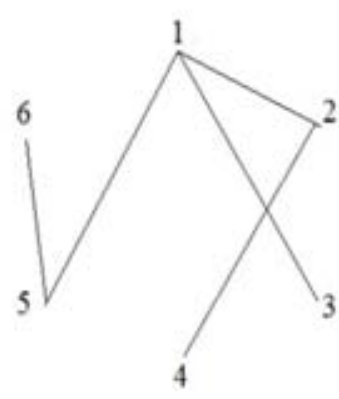

a. Original Graph

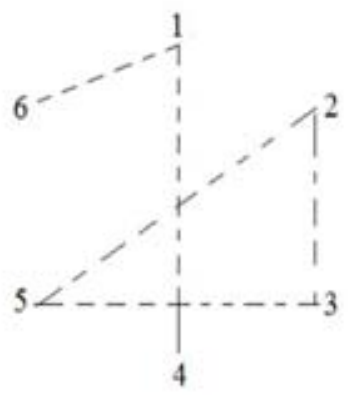

b. Two-Hop Graph

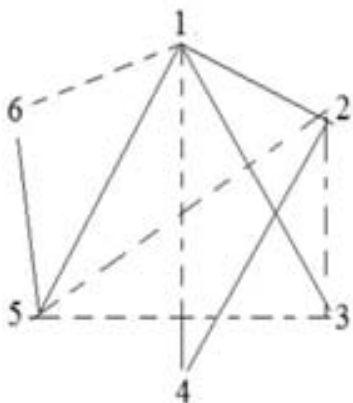

c. Joint Graph

Figure 1: (a) an undirected graph, (b) its Two-Hop graph, and (c) their Joint graph.

Fig 1 illustrates how the second-order neighbour could help increase the consensus speed. The two-hop graph (Fig la) consists of the second order neighbours' connections for each node. For instance, node 1 has a second order neighbour of node 6 through node 5, and node 4 through node 2 . A consensus protocol including the information of both first and second order neighbours would add a virtual two-hop graph to the original graph, and give the joint graph topology as shown in Fig lc. As it can be seen, this graph has more connections and therefore a higher algebraic connectivity, which leads to a faster consensus speed. Note that one node can be both a first order neighbour and a second order neighbour; the information on that node will be used twice during consensus if this is the case. 
It is known that the consensus protocol involving second order neighbours can effectively increase the consensus speed, in which [3] and [4] analysed the performance of such a protocol. However we cannot apply nearest neighbour coefficient setting method directly to the two-hop protocol, since the constraint on $a_{i j}$ is only derived for first order neighbour consensus. It can be easily verified that the Uniform method would guarantee the convergence, however it has fixed weights, which are proven to have a slower consensus speed compare to the adaptive coefficient setting method. Therefore we look for an adaptive coefficient setting method similar to the Metropolis method but involving the information from second order neighbours.

Murray and Jin described the two-hop protocol in [3] as the following:

$x_{i}^{t+1}=x_{i}^{t}+\sum_{j \in N_{i}} a_{i j}\left(x_{j}^{t}-x_{i}^{t}\right)+\sum_{k \in N_{i}^{2}} w_{i k}\left(x_{k}^{t}-x_{i}^{t}\right)$

\section{Adaptive coefficient setting method for two-hop consensus}

We propose the following two-hop consensus protocol inspired by the Metropolis and Mean Metropolis method, the proof of convergence of this protocol is provided in the Appendix.

$$
\begin{aligned}
& x_{i}^{t+1}=x_{i}^{t}+\frac{1}{2} \sum_{j \in N_{i}} a_{i j}\left(x_{j}^{t}-x_{i}^{t}\right)+\frac{1}{2} \sum_{k \in N_{i}^{2}} w_{i k}\left(x_{k}^{t}-x_{i}^{t}\right) \\
& \text { where } \quad a_{i j}=\left\{\begin{array}{cc}
\frac{2 \sqrt{2}}{N_{i}+N_{j}+N_{i}^{2}+N_{j}^{2}-1}, & v_{j} \in N_{i} \\
-\sum_{j \in N_{i}} \frac{2 \sqrt{2}}{N_{i}+N_{j}+N_{i}^{2}+N_{j}^{2}-1}, & i=j \\
0, & \text { otherwise }
\end{array}\right. \\
& w_{l k}=\left\{\begin{array}{cc}
\sum_{j \in N_{i}} a_{i j} a_{j k}, \quad v_{k} \in N_{i}^{2} & \\
-\sum_{k \in N_{i}^{2}} \sum_{j \in N_{i}} a_{i j} a_{j k}, & i=k \\
0, & \text { otherwise }
\end{array}\right.
\end{aligned}
$$

where $x_{i}^{t}, x_{i}^{t+1}$ represent the information of $\mathrm{i}^{\text {th }}$ agent at $\mathrm{t}^{\text {th }}$ and $(\mathrm{t}+\mathrm{l})^{\text {th }}$ iteration respectively.

\section{Updating scheme}

We have mentioned that the discrete time first order neighbour protocol has a matrix representation of $X^{k+1}=D X^{k}$. The updating scheme for two-hop consensus is derived to be $X^{k+1}=\left(I+A^{\prime}+2 W^{\prime}\right) X^{k}$, 
where $a_{i j}^{\prime}=\frac{a_{i j}}{2}, w_{i k}^{\prime}=\frac{w_{i k}}{2}$ such that $\mathrm{A}=2 \mathrm{~A}^{\prime}, \mathrm{W}=2 \mathrm{~W}^{\prime}$

This scheme is implemented in our simulation. The setting of our simulation is undirected fixed topology of linear average consensus protocol in discrete time. The marginal error at consensus is set to $1 \%$ of the average initial value of the nodes within the network, that is, the information collection process will terminate once the following inequality is satisfied:

$$
\sum_{i=1}^{n-1}\left|x_{i}^{e}-x_{i+1}^{\mathrm{f}}\right|<1 \% \times \frac{1}{n} \sum_{i=1}^{n} x_{i}^{0}
$$

This error should be designed differently for other applications. Bearing in mind that in real world applications, the error function needs to be distributed, and should depend on the scale of initial values of nodes within the system. The smaller this error is, the longer the consensus time will be.

\section{Simulation results}

In the first part of the investigation, we look at how the graph topology affects the consensus speed, and from this, we suggest network layout for the power systems. It was shown that the algebraic connectivity $\left|\lambda_{2}\right|$ of graph laplacian reflects the consensus speed in [7]. In order to maximize the consensus speed, the updating protocol needs to be properly designed. [5] has shown that the Mean Metropolis method can achieve near optimal consensus speed, and the optimal speed can be obtained by designing a D with minimum second largest eigenvalue, denoted as $\left|\lambda_{2}^{\prime}\right|$. Therefore we will use the Mean Metropolis method to set the coefficients, with ideal epsilon found by Matlab such that $\left|\lambda_{2}^{\prime}\right|$ is minimized. We have collected simulation results for tree, star, ring, two-hop and complete topologies, with number of nodes ranging from 6 up to 100.

\section{Table 1: Comparison of $\left|\lambda_{2}^{\prime}\right|$ for Different Graph Topology}

\begin{tabular}{|l|l|l|l|l|l|}
\hline \multirow{2}{*}{$\begin{array}{l}\text { Number of } \\
\text { nodes }\end{array}$} & \multicolumn{4}{|l}{ Second Smallest Eigenvalue for Different Graph Topology } \\
\cline { 2 - 6 } & Tree & Star & Ring & Two-Hop & Complete \\
\hline 6 & 0.8592 & 0.7147 & 0.6008 & 0.2008 & 0.0008 \\
\hline 7 & 0.8937 & 0.7503 & 0.6705 & 0.3232 & 0.0007 \\
\hline 8 & 0.9147 & 0.7780 & 0.7443 & 0.3980 & 0.0006 \\
\hline$\ldots$ & $\ldots$ & $\ldots$ & $\ldots$ & $\ldots$ & $\ldots$ \\
\hline 100 & 0.9957 & 0.9802 & 0.9980 & 0.9951 & 0 \\
\hline $\begin{array}{l}\text { Number of } \\
\text { connections } \\
\text { for N nodes }\end{array}$ & $\mathrm{N}-1$ & $\mathrm{~N}-1$ & $\mathrm{~N}$ & $2 \mathrm{~N}$ & $\mathrm{~N}(\mathrm{~N}-1) / 2$ \\
\hline
\end{tabular}


We then repeatedly ran the simulations to compare the consensus speed of the first order neighbour protocol using the Metropolis method $X^{k+1}=D X^{k}$, with our proposed coefficient setting method for two-hop protocol $X^{k+1}=\left(I+A^{\prime}+\right.$ $\left.2 W^{\jmath}\right) X^{k}$.

\section{Table 2: Comparison of Consensus Speed using Different Protocols}

\begin{tabular}{|c|c|c|c|c|}
\hline \multirow{2}{*}{$\begin{array}{l}\text { Number } \\
\text { of Nodes }\end{array}$} & \multirow{2}{*}{$\begin{array}{l}\text { Average Number of } \\
\text { Connections per Node }\end{array}$} & \multicolumn{2}{|c|}{ Number of Iterations Required } & \multirow{2}{*}{$\begin{array}{l}\text { Percentage Speed } \\
\text { Increase }\end{array}$} \\
\hline & & $\begin{array}{l}\text { Nearest Neighbour - } \\
\text { Metropolis Method }\end{array}$ & Two-Hop & \\
\hline 7 & 0.86 & 82 & 36 & $128 \%$ \\
\hline 7 & 1.42 & 23 & 15 & $53 \%$ \\
\hline 15 & 0.93 & 338 & 202 & $67 \%$ \\
\hline 15 & 1.33 & 110 & 83 & $33 \%$ \\
\hline 30 & 0.97 & 1729 & 974 & $78 \%$ \\
\hline 30 & 1.33 & 193 & 149 & $30 \%$ \\
\hline 30 & 1.70 & 64 & 51 & $25 \%$ \\
\hline 60 & 0.98 & 4928 & 2474 & $99 \%$ \\
\hline 60 & 1.30 & 501 & 340 & $47 \%$ \\
\hline 60 & 1.50 & 256 & 207 & $24 \%$ \\
\hline
\end{tabular}

\section{Discussion}

There are many potential applications of consensus protocols; we will only focus on power system implementations. In power systems, once a fault node has been cleared, it is necessary to restore the out-of-service but un-faulted node as fast as possible to maintain supply, this is the load restoration process. Also if there is insufficient power generation within the system, it is necessary to intelligently shed some load in order to obtain demand-supply balance, this is the load shedding process. Both load restoration and load shedding processes require the application of consensus protocols in order to obtain the global information distributedly, including those that address which node has failed, which node has been affected and what is the net power of the system. By increasing the consensus speed, the power system will have a better performance and a faster response. There are two obvious factors that have a significant impact on the consensus speed: one is the graph topology, i.e. the layout of network agents; and the other is consensus protocol, i.e. how the consensus proceeds. We will therefore look at how to improve the consensus speed in those two ways. 


\section{The effect of graph topology}

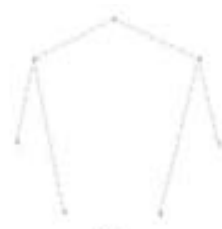

a

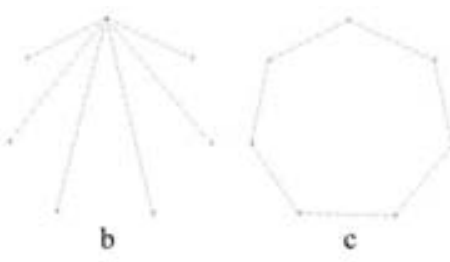

b

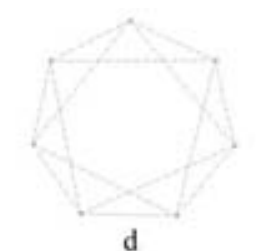

d

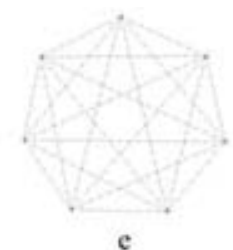

e

Figure 2: Different graph topologies are (a) Tree, (b) Star, (c) Ring, (d) Two-Hop, and (e) Complete.

For each topology, the second largest eigenvalue of weighted graph laplacian is calculated. It can be easily observed in Table 1 that complete topology (Fig 2e) has the fastest consensus speed, each with an $\left|\lambda_{2}^{\prime}\right|$ almost zero. In fact, the system will reach consensus after one step for nodes number $\geq 12$. However, in real world power systems, building a connection between every pair of nodes to get a complete graph topology is not feasible.

Tree (Fig 2a), star (Fig 2b) and ring (Fig 2c) topologies for $\mathrm{N}$ nodes will have $\mathrm{N}-1, \mathrm{~N}-1$ and $\mathrm{N}$ connections, respectively; while two-hop (Fig 2d) has $2 \mathrm{~N}$ connections. Although two-hop topology has a faster consensus speed than tree and ring topology, in terms of connection-cost it appears to be inefficient. This topology also has a slower speed than star topology in large networks, therefore the two-hop topology may not be suitable in real world power systems.

Tree, star and ring topologies have almost the same number of connections for the same number of nodes. For smaller networks (less than 10 nodes), ring topology has the fastest speed, while star topology is more preferable in large networks. It was simulated that for a 100-node system, the time required to reach consensus for ring topology is ten times more than the time required for star topology. A possible explanation for such behaviour is that in a small network the distance between any pair of nodes in ring topology is short; while in large networks, the distance is too long. The advantage of star topology, on the other hand, is that the maximum distance between any pair of two nodes is two connections in length, and every other node is directly connected with a central node such that the nodes can communicate more efficiently.

It is important to note that the communication structure is independent of the physical infrastructure of Smart Grid or other power systems. The communication between two neighbouring nodes can still carry on even if the physical distribution line is disconnected. Therefore, the graph topologies discussed in this report refer to the communication structure and not the actual physical structures. 


\section{The effect of consensus protocol}

Since the two-hop protocol is the equivalent of adding a virtual two-hop graph to the original graph, resulting in a joint graph, we simulate the consensus process for the joint graph but using first-order neighbour protocol. This simulation would keep the communication cost the same, as the controlled variable. Results show that the consensus speed for the joint graph using first order neighbour protocol is faster. However it does not indicate that two-hop protocol is a bad choice. Since we have used the actual joint graph in the simulation, this means that there are more actual connections between nodes rather than the virtual connections in two-hop protocol, which would increase the infrastructure cost of the network.

The simulations were performed repeatedly to compare the consensus speed of first order neighbour protocol using the Metropolis method with our proposed coefficient setting method for two-hop protocol. Table 2 demonstrates that the increase in consensus speed varies under different graph topologies. There appears to be a trend such that the more connections there are, the less effective the two-hop protocol. An explanation for this trend is that if the original graph already has many connections, then the two-hop graph is only going to add fewer new connections, such that the increase in consensus speed is smaller. Nevertheless, we can see that from our simulation, there is a $24 \%$ to $128 \%$ increase in consensus speed by using our proposed protocol.

However, by involving a second-order neighbour into the consensus, extra information needs to be communicated between the agents through network connections. It was shown in [3] that the bandwidth requirement is doubled compare to the first order neighbour protocol. Also, employment of the two-hop protocol results in additional time delay in communication as the second order neighbours need to first pass their information to the first order neighbours.

\section{Conclusion}

We reviewed consensus protocols, some of their applications, and discussed different forms of existing protocols. Based on our Matlab simulation, we found that the star topology gives the fastest consensus speed for large networks with the consideration of communication cost. Ring and two-hop topologies have better performance for smaller networks. Complete graph un-doubtfully gave the fastest consensus speed, but the communication cost is extremely high. We slightly modified the original two-hop protocol and proposed an adaptive coefficient setting method inspired by the Metropolis method, verified its convergence and also derived the matrix form of the updating scheme for our 
two-hop protocol. It is able to deliver a faster consensus speed and the simulation shows that the speed increased up to $100 \%$. However there are some trade-offs of implementing the two-hop protocol, such as extra bandwidth requirement and communication time delay.

Our proposed adaptive coefficent setting method for two-hop protocol will increase the consensus speed, and save a large proportion of time during the consensus process. In the case of power systems such as Smart Grid, this will reduce the time taken for actions such as load restoration and load shedding, therefore saving power and increasing the overall efficiency of the system.

\section{Acknowlegement}

I would like to thank Jiahu Qin and Dr. Brad Yu for fruitful discussions and their advice on this project.

\section{Bibliography}

[1] R. Olfati-Saber, "Flocking for multi-agent dynamic systems: algorithms and theory," Automatic Control, IEEE Transactions on, vol. 51, pp. 401-420, 2006.

[2] W. Ren, "Synchronization of coupled harmonic oscillators with local interaction," Automatica, vol. 44, pp. 3195-3200, 2008.

[3] J. Zhipu and R. M. Murray, "Multi-Hop Relay Protocols for Fast Consensus Seeking," in Decision and Control, 2006 45th IEEE Conference on, 2006, pp. 1001-1006.

[4] D. Yuan, et al., "Accelerating distributed average consensus by exploring the information of second-order neighbors," Physics Letters A, vol. 374, pp. 2438-2445, 2010.

[5] X. Yinliang and L. Wenxin, "Novel Multiagent Based Load Restoration Algorithm for Microgrids," Smart Grid, IEEE Transactions on, vol. 2, pp. 152-161, 2011.

[6] A. Jadbabaie, et al., "Coordination of groups of mobile autonomous agents using nearest neighbor rules," Automatic Control, IEEE Transactions on, vol. 48, pp. 988-1001, 2003. 
[7] R. Olfati-Saber and R. M. Murray, "Consensus problems in networks of agents with switching topology and time-delays," Automatic Control, IEEE Transactions on, vol. 49, pp. 1520-1533, 2004.

[8] R. Wei and R. W. Beard, "Consensus seeking in multiagent systems under dynamically changing interaction topologies," Automatic Control, IEEE Transactions on, vol. 50, pp. 655-661, 2005.

[9] R. Olfati-Saber, et al., "Consensus and Cooperation in Networked MultiAgent Systems," Proceedings of the IEEE, vol. 95, pp. 215-233, 2007.

[10]X. Yinliang, et al., "Stable Multi-Agent-Based Load Shedding Algorithm for Power Systems," Power Systems, IEEE Transactions on, vol. 26, pp. 20062014, 2011.

[11]"Technology Road Map, Smart Grids," International Energy Agency, 2011.

[12] C.W.Reynolds, "Flocks, herds, and schools: A distributed behavioral model," in ACM SIGGRAPH, 1987, pp. 25-34.

[13]S. Steven H, "From Kuramoto to Crawford: exploring the onset of synchronization in populations of coupled oscillators," Physica D: Nonlinear Phenomena, vol. 143, pp. 1-20, 2000.

[14] D. P. Spanos, et al., "Approximate distributed Kalman filtering in sensor networks with quantifiable performance," in Information Processing in Sensor Networks, 2005. IPSN 2005. Fourth International Symposium on, 2005, pp. 133-139.

[15]X. Lin, Stephen Boyd, Seung-Jean Kim, "Distributed Average Consensus with Least-Mean-Square Deviation," in Proceedings of the 17th International Symposium on Mathematical Theory of Networks and Systems, Kyoto, Japan, 2006.

[16]J. Kennedy and R. Eberhart, "Particle swarm optimization," in Neural Networks, 1995. Proceedings., IEEE International Conference on, 1995, pp. 1942-1948 vol.4. 


\section{Appendix}

Proof of Convergence for the Proposed Adaptive Coefficient Setting Method

$$
\begin{gathered}
x_{i}^{i+1}=x_{i}^{t}+\frac{1}{2} \sum_{j \in N_{i}} a_{i j}\left(x_{j}^{t}-x_{i}^{t}\right)+\frac{1}{2} \sum_{k \in N_{i}^{2}} w_{i k}\left(x_{k}^{t}-x_{i}^{t}\right) \\
\text { where } \quad a_{i j}\left\{\begin{array}{cc}
\frac{2 \sqrt{2}}{N_{i}+N_{j}+N_{i}^{2}+N_{j}^{2}-1}, & v_{j} \in N_{i} \\
-\sum_{j \in N_{i}} \frac{2 \sqrt{2}}{N_{i}+N_{j}+N_{i}^{2}+N_{j}^{2}-1}, & i=j \\
w_{i k}= & \left\{\begin{array}{cc}
\sum_{j \in N_{i}} a_{i j} a_{j k}, & v_{k} \in N_{i}^{2}
\end{array}\right. \\
-\sum_{k \in N_{i}^{2}} \sum_{j \in N_{i}} a_{i j} a_{j k}, & i=k \\
0, & \text { otherwise }
\end{array}\right.
\end{gathered}
$$

where $x_{t^{\prime}}^{i} x_{t+1}^{i}$ represent the information of the $\mathrm{i}^{\text {th }}$ agent at the $\mathrm{t}^{\text {th }}$ and $(\mathrm{t}+1)^{\text {th }}$ iteration respectively.

We can multiply both sides of (1) by two and split it into (4) and (5):

$$
\begin{aligned}
& x_{i}^{t+1}=x_{i}^{t}+\sum_{j \in N_{i}} a_{i j}\left(x_{j}^{t}-x_{i}^{t}\right) \\
& x_{i}^{t+1}=x_{i}^{t}+\sum_{k \in \mathbb{N}_{i}^{2}} w_{i k}\left(x_{k}^{t}-x_{i}^{t}\right)
\end{aligned}
$$

It was shown in [5] that the stability of the first order neighbour consensus protocol can be guaranteed provided that

$$
0<a_{i j}<\frac{2}{\left(N_{i}+N_{j}\right)}
$$

If we only consider the two-hop graph, then $w_{i k}$ is also a weighted Laplacian for the same graph, we can conduct a stability analysis using exactly the same derivation in $[5,10]$, where by defining a Lyapunov function as the following $V^{t}=\left(X^{t}\right)^{\mathrm{T}} X^{t}, \Delta V=V t^{+1}-V^{t}$ the stability can be guaranteed provided that:

$0<w_{i k}<\frac{2}{\left(N_{i}+N_{k}\right)}$

In order for (1) to converge, we show both (4) and (5) converge individually, and as a result the sum of two convergence protocols also converge. We firstly show that the new $a_{i j}$ satisfies condition (6), such that (4) will converge. 
The ANU Undergraduate Research Journal

$$
\begin{aligned}
a_{i j} & =\frac{2 \sqrt{2}}{N_{i}+N_{j}+N_{i}^{2}+N_{j}^{2}-1} \\
& <\frac{2 \sqrt{2}}{N_{i}+N_{j}+N_{j}+N_{i}-1} \\
& =\frac{\sqrt{2}}{N_{i}+N_{j}-\frac{1}{2}}<\frac{2}{N_{i}+N_{j}}
\end{aligned}
$$

Substitution used: $N_{i}^{2} \geq N_{j}, N_{j}^{2} \geq N_{i} . \quad N_{i}+N_{j} \geq 3$

Secondly, we show that the new weight $w_{i k}$ satisfies (7) such that (5) will converge.

$$
\begin{aligned}
w_{i k} & =\sum_{j \in V} a_{i j} a_{j k}=\sum_{j \in V} \frac{8}{\left(N_{i}+N_{j}+N_{i}^{2}+N_{j}^{2}-1\right)\left(N_{j}+N_{k}+N_{j}^{2}+N_{k}^{2}-1\right)} \\
& \leq \frac{4\left(N_{i}+N_{k}\right)}{\left(N_{i}+\min \left[N_{j}\right\}+N_{i}^{2}+\min \left\{N_{j}^{2}\right]-1\right)\left(\min \left\{N_{j}\right\}+N_{k}+\min \left\{N_{j}^{2}\right]+N_{k}^{2}-1\right)} \\
& \leq \frac{4\left(N_{i}+N_{k}\right)}{\left(N_{i}+N_{i}^{2}+N_{i}+N_{k}-1\right)\left(N_{k}+N_{k}^{2}+N_{i}+N_{k}-1\right)} \\
& \leq \frac{4\left(N_{i}+N_{k}\right)}{\left(2 N_{i}+N_{k}\right)\left(N_{i}+2 N_{k}\right)}=\frac{4\left(N_{i}+N_{k}\right)}{2\left(N_{i}+N_{k}\right)^{2}+N_{i} N_{k}} \\
& \leq \frac{4\left(N_{i}+N_{k}\right)}{2\left(N_{i}+N_{k}\right)^{2}}=\frac{2}{\left(N_{i}+N_{k}\right)} \quad
\end{aligned}
$$

Since our proposed coefficients $a_{i j}$ and $w_{i k}$ satisfy the conditions (6) and (7) respectively, both (4) and (5) converge such that the overall protocol (1) will also converge. 